05,13

\title{
Условия возникновения спонтанной осциллирующей магнитной релаксации в синтетических ферримагнетиках $\mathrm{Pt} / \mathrm{Co} / \mathrm{Ir} / \mathrm{Co} / \mathrm{Pt}$
}

\author{
() Р.Б. Моргунов ${ }^{1,2,3}$, А.И. Безверхний ${ }^{1,2}$, О.С. Дмитриев ${ }^{2}$, М.В. Бахметьев ${ }^{1}$ \\ ${ }^{1}$ Институт проблем химической фризики РАН, \\ Черноголовка, Россия \\ 2 Тамбовский технический государственный университет, \\ Тамбов, Россия \\ ${ }^{3}$ Первый МГМУ им. И.М. Сеченова Минздрава России (Сеченовский университет), \\ Москва, Россия \\ E-mail: morgunov2005@yandex.ru
}

Поступила в Редакцию 9 ноября 2019 г.

В окончательной редакции 9 ноября 2019 г.

Принята к публикации 14 ноября 2019 г.

\begin{abstract}
Установлено, что немонотонная релаксация намагниченности в синтетических ферримагнетиках (СФ) c перпендикулярной магнитной анизотропией $\mathrm{Pt} / \mathrm{Co} / \mathrm{Ir} / \mathrm{Co} / \mathrm{Pt}$ вызвана совпадением двух или трех полей переключения между стабильными состояниями намагниченности СФ (двойная или тройная точка). Поля переключения между стабильными состояниями СФ определяются соотношением зеемановской, межслоевой обменной энергии и энергий барьеров перемагничивания каждого слоя, зависящих от толщин слоев и температуры. Показано, что подбор толщины и/или температуры позволяет достигать двойных и тройных точек, в которых поля переходов между состояниями совпадают, что вызывает немонотонную магнитную релаксацию СФ.
\end{abstract}

Ключевые слова: синтетический ферримагнетик, перпендикулярная магнитная анизотропия, релаксация намагниченности, обменное взаимодействие.

DOI: 10.21883/FTT.2020.03.49005.583

\section{1. Введение}

Синтетические ферримагнетики (СФ) с перпендикулярной магнитной анизотропией состоят из двух слоев ферромагнетика разной толщины $(\sim 1 \mathrm{~nm})$ разделенных тонким слоем немагнитного материала (спейсера) (толщина $\sim 1 \mathrm{~nm}$ ), который обеспечивает обменное взаимодействие RKKY (Ruderman-Kittel-Kasuya-Yosida) между ферромагнитными слоями, достаточно слабое, чтобы их намагниченности можно было переключать внешним магнитным полем. Хотя первоначальный интерес к таким структурам был связан с наличием в них гигантского магнитосопротивления, впоследствии выяснилось, что это не единственная причина и способ применения СФ в спинтронике. Исследуемые в данной работе СФ интересны наличием асимметричного обменного взаимодействия Дзялошинского-Мория на интерфейcax $\mathrm{Co} / \mathrm{Pt}$ и $\mathrm{Co} / \mathrm{Ir}$, где взаимодействие ДзялошинскогоМория генерирует спиновые неоднородности - скирмионы, управляемые спин-поляризованным током. Поэтому, хотя магнитосопротивление исследуемых структур не превышает $1 \%$, они являются перспективными устройствами хранения данных на основе магнитных солитоноподобных образований (скирмионов). Взаимодействие Дзялошинского-Мория возникает при отсутствии центра инверсии на интерфейсе. Оно расщепляет вырожденные правосторонние и левосторонние повороты намагниченности в доменной стенке.
Поскольку обменное взаимодействие RKKY в СФ приводит к взаимодействию слоев, а на интерфейсе к нему добавляется асимметричное взаимодействие Дзялошинского-Мория, можно ожидать обнаружения новых закономерностей движения доменных стенок и процесса перемагничивания таких структур.

СФ имеют четыре стабильных состояния намагниченности: два состояния с параллельной ориентацией намагниченности в слоях ферромагнетика $\left(P^{+}-\uparrow \uparrow, P^{-}-\downarrow \downarrow\right)$ и два с антипараллельной $\left(A P^{+}-\downarrow \uparrow, A P^{-}-\uparrow \downarrow\right)$. Рaнее нами были обнаружены немонотонная [1] и осциллирующая релаксации намагниченности длительностью $\sim 40 \mathrm{~min}$ (рис. $1, a, b)[2]$ в $\mathrm{Pt} / \mathrm{Co} / \mathrm{Ir} / \mathrm{Co} / \mathrm{Pt}$ в постоянном магнитном поле. Попытка их описания при помощи модели макроспина [1] дала лишь качественное сходство результатов моделирования с экспериментом. Это объясняется тем, что модель макроспина не учитывает существование доменной структуры, а перемагничивание происходит во всем слое ферромагнетика одновременно.

Как видно из рис. $1, a, b)$, при немонотонной релаксации система переходит из состояния $A P^{+}$в более выгодное по энергии $A P^{-}$через промежуточное состояние $P^{-}$. Немонотонная и осциллирующая релаксация в СФ наблюдается в условиях совпадения критических полей переходов между тремя стабильными состояниями намагниченности, т.е. при достижении $H_{\mathrm{C}}$ (рис. 2). Стартовые условия для переходов между состояниями определяются балансом Зеемановской, обменной 
энергии и энергии барьеров перемагничивания. Величина энергетического барьера перемагничивания зависит от механизма перемагничивания и выражается как $E_{B}=K_{A} V_{a}$, где $K_{A}$ - константа магнитной анизотропии, $V_{a}$ - активационный объем. В зависимости от механизма перемагничивания будет меняться и активационный объем:

1. При перемагничивании путем когерентного вращения намагниченности $V_{a}$ будет равен объему всего образца.

2. При перемагничивании путем множественного зародышеобразования $V_{a}$ будет равен объему зародыша.

3. При перемагничивании путем движения доменной стенки, активационный объем зависит от концентрации дефектов, на которых закрепляется доменная стенка,
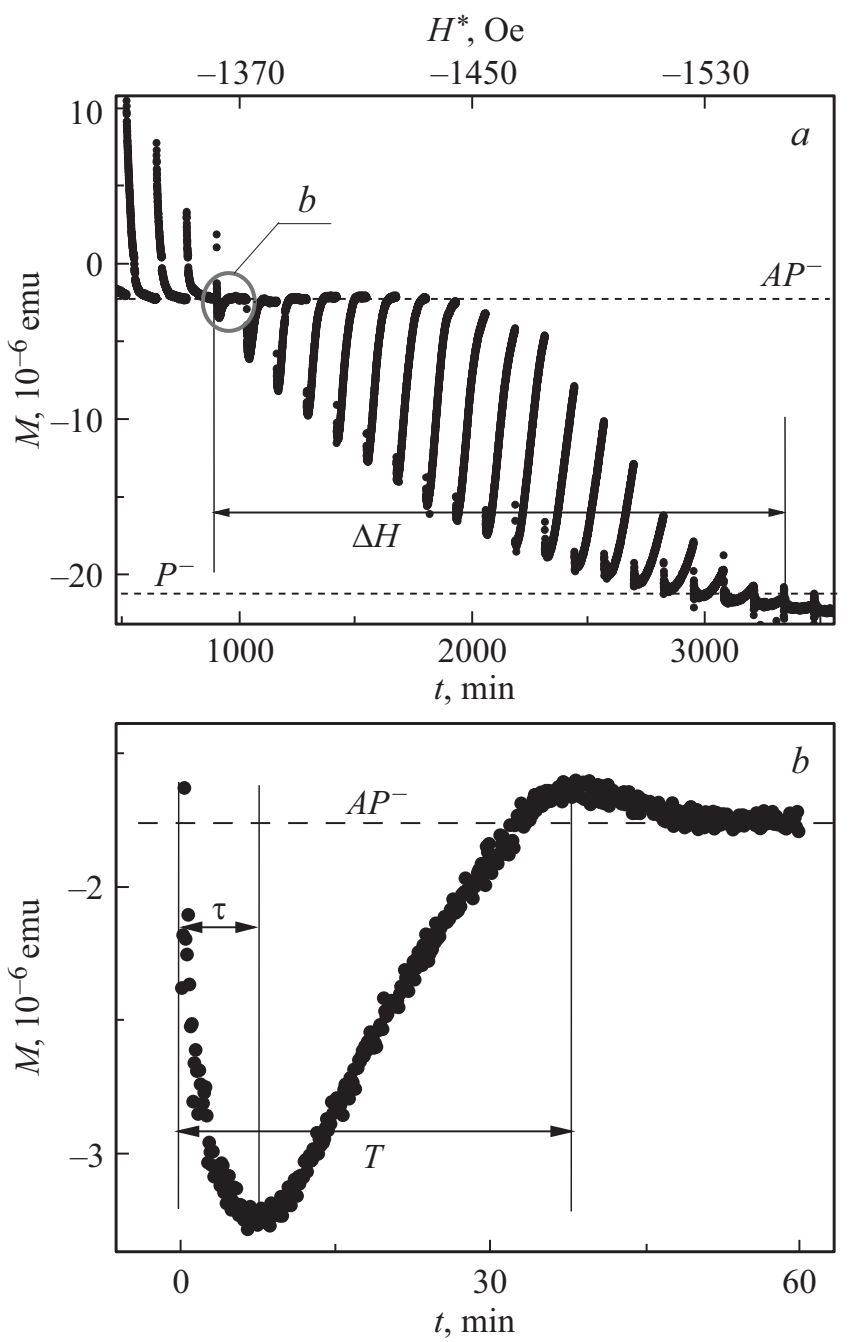

Pис. 1. a) Набор кривых релаксации намагниченности при $T=100 \mathrm{~K}$ в постоянных полях $H^{*}$. Горизонтальными прерывистыми линиями показаны стабильные состояния намагниченности $A P^{-}$и $P^{-}$. Интервал полей, в котором наблюдается немонотонная релаксация, обозначен $\Delta H . b)$ Выделенная на рис. $1, a$ релаксация намагниченности в постоянном магнитном поле -1370 Ое. $\tau$ обозначено время от начала релаксации до глобального минимума намагниченности.

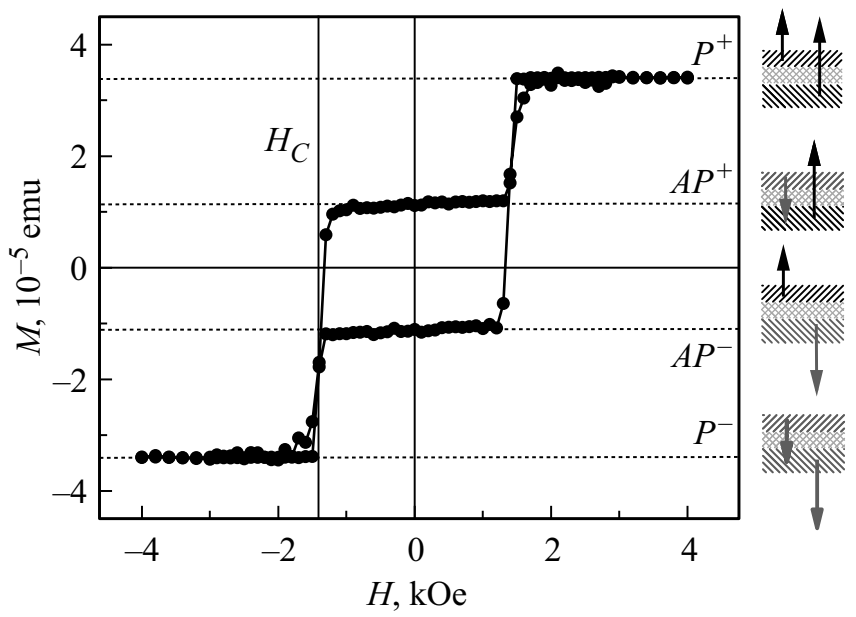

Рис. 2. Полевая зависимость намагниченности при температуре $T=100 \mathrm{~K}$. Горизонтальными прерывистыми линиями показаны стабильные состояния намагниченности $P^{+}, A P^{+}$, $A P^{-}$и $P^{-}$, а на боковых ставках показаны схемы поясняющие направления намагниченности в слоях Со для каждого состояния. Вертикальной линией $H_{\mathrm{C}}$ указанно поле перехода между состояниями $A P^{+}$и $P^{-}$и между $P^{-}$и $A P^{-}$.

от параметров дефектов, температуры, намагниченности насыщения образца и флуктуационного поля.

Целью данной работы является установление количественного критерия возникновения немонотонной релаксации намагниченности, который позволял бы предсказывать экспериментальные условия для наблюдения такой релаксации в синтетических ферримагнетиках с перпендикулярной магнитной анизотропией.

\section{2. Методика}

В опытах использовали многослойную гетероструктуру $\mathrm{SiO}_{2} / \mathrm{Pt}(3.2 \mathrm{~nm}) / \mathrm{Co}(1.1 \mathrm{~nm}) / \mathrm{Ir}(1.4 \mathrm{~nm}) / \mathrm{Co}(0.7 \mathrm{~nm}) /$ $\mathrm{Pt}(3.2 \mathrm{~nm})$ площадью $4 \times 5 \mathrm{~mm}^{2}$, приготовленную методом магнетронного напыления при комнатной температуре, аналогично условиям, описанным в [3]. Толщины слоев определялись исходя из времени их напыления. Аттестация магнитных свойств образца приведена в [4]. Перпендикулярная магнитная анизотропия (ПМА) обусловлена гибридизацией $3 d$ орбитального момента Со с $5 d$ орбитальным моментом Pt и $\operatorname{Ir}$ [5]. При используемых толщинах слоев Co $<2 \mathrm{~nm}$ поверхностная перпендикулярная магнитная анизотропия доминирует над объемной анизотропией. Измерение релаксации намагниченности и полевых зависимостей намагниченности производились СКВИД-магнитометром MPMS 5XL Quantum Design. Величины намагниченностей насыщения слоев и их удельные энергии барьеров перемагничивания при разных температурах были определены нами в [6].

Для измерения релаксации намагниченности устанавливалось поле, превышающее поле насыщения образца $(4 \mathrm{kOe})$. После насыщения напряженность поля меня- 
лась на значение $H^{*}$, близкое к полю переключения межу состояниями $A P^{+}$и $P^{-}$, или между состояниями $P^{-}$и $A P^{-}$(рис. 2 , вертикальная линия $H_{\mathrm{C}}$ ). Далее, сразу после переключения поля, измерялось изменение намагниченности образца с течением времени в постоянном магнитном поле. Данная процедура повторялась для различных значений поля $H^{*}$. Петли магнитного гистерезиса были записаны в диапазоне температур от 50 до $300 \mathrm{~K}$ с шагом в $25 \mathrm{~K}$. Скорость развертки при этом была $0.2 \mathrm{Oe} / \mathrm{s}$, что можно назвать квазистатическим режимом, поскольку эффекты, связанные со скоростью развертки поля [7], при этом не проявлялись.

Для определения скорости движения доменной стенки образец насыщался в поле (out-of-plane) $H_{\mathrm{OP}}=+800 \mathrm{Oe}$, направленном перпендикулярно к поверхности образца, после чего поле $H_{\mathrm{OP}}$ менялось на значение, близкое к полю переключения между стабильными состояниями намагниченности. В этом постоянном поле велась запись МОКЕ снимков поверхности с интервалом $0.6 \mathrm{~s}$ при помощи микроскопа на основе магнитооптического полярного эффекта Kерра Durham Magneto-optics NanoMOKE3. Данные измерения производились при $T=300 \mathrm{~K}$.

Для установления наличия взаимодействия Дзялошинского-Мория использовался метод асимметричного расширения магнитных доменов [8,9], при помощи микроскопа на основе магнитооптического полярного эффекта Keppa Durham Magneto-optics NanoMOKE3. Для наблюдения асимметричного расширения магнитных доменов образец насыщался при $T=300 \mathrm{~K}$ в постоянном магнитном поле $H_{\mathrm{OP}}=+800 \mathrm{Oe}$, после чего значение этого поля менялось на $H_{\mathrm{OP}}=-380$ Ое. Одновременно с переключением поля $H_{\mathrm{OP}}$ прикладывалось поле (inplane) $H_{\mathrm{IP}}$, направленное в плоскости образца, как показано на рис. 3 , и велась запись снимков поверхности с интервалом $0.6 \mathrm{~s}$. Процедура проводилась для значений поля $H_{\mathrm{iP}}=0,50$ и $100 \mathrm{Oe}$.

\section{3. Результаты и обсуждение}

Немонотонная релаксация намагниченности наблюдалась в полях, близких к полю переключения между тремя стабильными состояниями намагниченности $H_{\mathrm{C}}$ (рис. 2). Совпадение полей переключения между состояниями $A P^{+} \rightarrow P^{-}$и $P^{-} \rightarrow A P^{-}$и между $A P^{-} \rightarrow P^{+}$ и $P^{+} \rightarrow A P^{+}$возникает у исследуемого образца при $T=100 \mathrm{~K}$. Влияние температуры на поля переходов между стабильными состояниями намагниченности СФ $\mathrm{Pt} / \mathrm{Co} / \mathrm{Ir} / \mathrm{Co} / \mathrm{Pt}$ подробно исследовано и проанализировано в [6]. Совпадение полей переключения между тремя стабильными состояниями намагниченности говорит о наличии на энергетической диаграмме состояний СФ в этом поле тройной точки, в которой могут существовать одновременно три магнитные фазы $\left(A P^{+}, A P^{-}, P^{-}\right.$в отрицательном поле, или $A P^{-}, A P^{+}, P^{+}-$в положительном). Одновременное наличие трех магнитных фаз

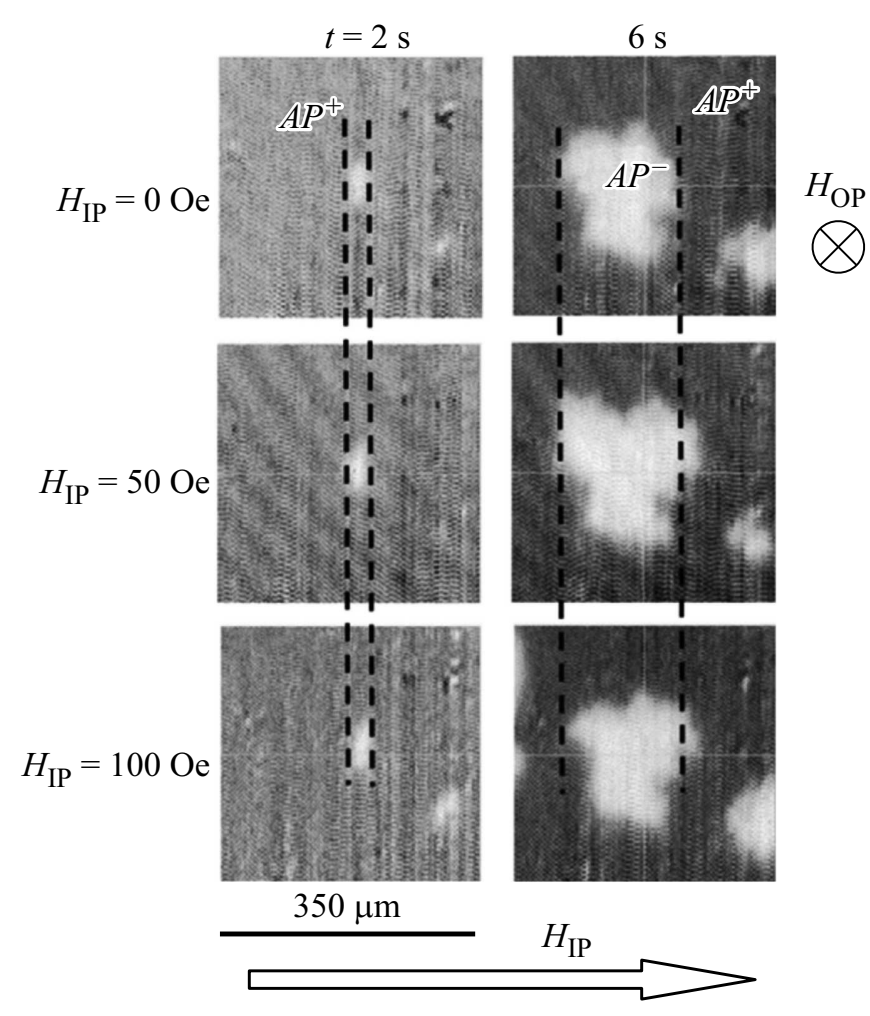

Рис. 3. Снимки, сделанные полярным микроскопом Керра при $T=300 \mathrm{~K}$, спустя 2 и $6 \mathrm{~s}$ после включения постоянного поля $H_{\mathrm{OP}}=-380$ Ое, в полях, $H_{\mathrm{IP}}=0,50$ и 100 Ое направленных вдоль плоскости пленки.

наблюдалось ранее в [7] для образца $\mathrm{SiO}_{2} / \mathrm{Pt}(3.2 \mathrm{~nm}) /$ $\mathrm{Co}(1.1 \mathrm{~nm}) / \mathrm{Ir}(1.4 \mathrm{~nm}) / \mathrm{Co}(1 \mathrm{~nm}) / \operatorname{Pt}(3.2 \mathrm{~nm})$ при $T=300 \mathrm{~K}$ методом микроскопии Керра.

Механизм магнитной релаксации для гетероструктур $\mathrm{Pt} / \mathrm{Co} / \mathrm{Ir} / \mathrm{Co} / \mathrm{Pt}$ был описан в [10] моделью FatuzzoLabrune $[11,12]$, которая учитывает два канала магнитной релаксации: множественное образование зародышей фазы обратной намагниченности и движение доменной стенки. В [10] релаксация исследовались при температуpax 200 и $300 \mathrm{~K}$, но параметр, отвечающий за перемагничивание путем движения доменной стенки, растет с понижением температуры для всех исследуемых образцов. Из этого можно сделать вывод о преимущественном вкладе в релаксацию, движения доменной стенки при $T=100 \mathrm{~K}$. Площадь исследуемого образца была значительно больше предела однодоменности тонкой пленки Со, поэтому поверхности энергетически выгодно разбиваться на магнитные домены, а релаксация не происходит путем когерентного поворота намагниченности всего слоя. Наличие магнитных доменов в образце при $T=300 \mathrm{~K}$ (рис. 3) доказывает, что при повышении температуры от 100 до $300 \mathrm{~K}$ перемагничивание осуществляется также путем движения доменной стенки.

Процесс релаксации был исследован при $T=300 \mathrm{~K}$ методом полярной микроскопии Керра в присутствии движущего поля $H_{\mathrm{OP}}=-380 \mathrm{Oе}$, направленного пер- 


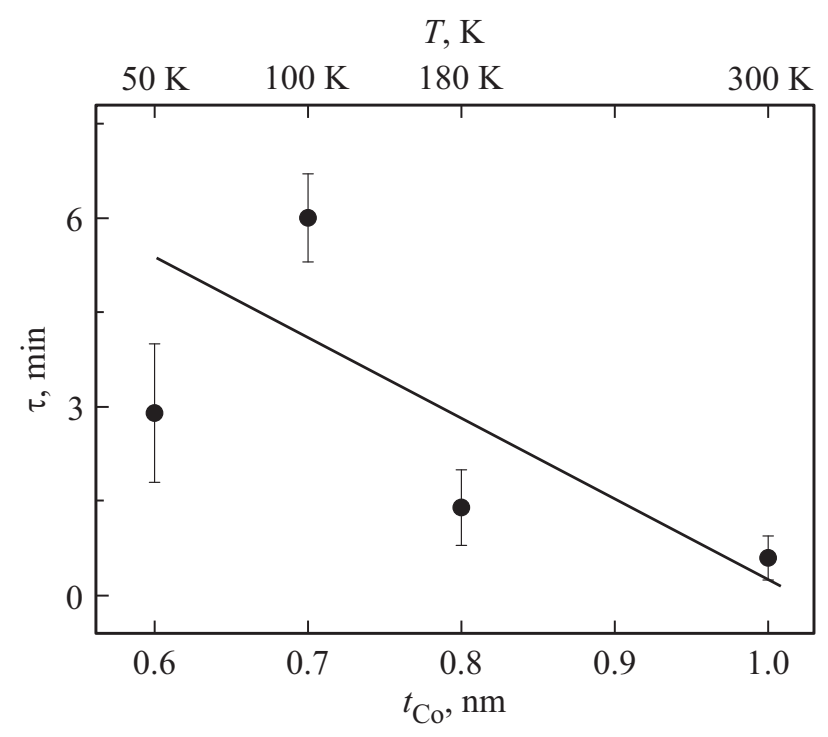

Рис. 4. Зависимость времени магнитной релаксации $\tau$, от толщины верхнего слоя Со $t_{\text {Со }}$ гетероструктуры $\mathrm{SiO}_{2} / \mathrm{Pt}(3.2 \mathrm{~nm}) / \mathrm{Co}(1.1 \mathrm{~nm}) / \mathrm{Ir}(1.4 \mathrm{~nm}) / \mathrm{Co}\left(t_{\mathrm{Co}}\right) / \mathrm{Pt}(3.2 \mathrm{~nm})$. Каждому образцу в соответствие ставится характерная температура $T^{*}$ (верхняя шкала), при которой в нем возникает немонотонная релаксация намагниченности.

пендикулярно поверхности образца, и в присутствии компоненты поля вдоль поверхности, как это показано на рис. 3. На рис. 3 видны магнитные домены, чьи правая и левая доменные границы движутся с разной скоростью, в зависимости от приложенного постоянного поля $H_{\mathrm{IP}}$. Асимметричное расширение магнитных доменов в таких условиях является доказательством наличия взаимодействия Дзялошинского-Мория $[8,9]$.

Осциллирующая релаксация намагниченности является частным случаем немонотонной релаксации, при котором амплитуда частичного перехода системы в состояние $A P^{+}$(маленький пик на рис. $\left.1, b\right)$ мала (меньше чувствительности СКВИД-магнитометра). Для количественного описания немонотонной релаксации было выбрано время $\tau$ (рис. $1, b$ ), за которое намагниченность релаксирует из начального состояния, в неравновесное состояние с минимальным значением намагниченности, но все еще не с минимальной энергией. Во всем диапазоне полей $\Delta H$ (рис. $1, a$ ), в котором наблюдается немонотонная релаксация, время $\tau$ было $\sim 6 \mathrm{~min}$. Мы сравнили время $\tau$ для образца, исследуемого в данной статье, со временем $\tau$ для других образцов данной серии, которые отличаются только толщиной верхнего слоя Со $\left(t_{\mathrm{Co}}=0.6,0.7,0.8,1.00 \mathrm{~nm}\right)$ (рис. 4). Из рис. 4 видно, что с увеличением толщины верхнего слоя Со, время $\tau$ имеет тенденцию к уменьшению. Поскольку в серии четыре образца, достоверность вышеуказанного утверждения невелика, но тенденция понятна, так как процесс перемагничивания является термоактивационным.

Для установления условий немонотонной релаксации необходимо сопоставить совпадение полей переходов между тремя стабильными состояниями с процессом немонотонной релаксации. Из рис. 1 видно, что при немонотонной релаксации система переходит из состояния $A P^{+}$в состояние $A P^{-}$через промежуточное состояние $P^{-}$, т.е. в постоянном магнитном поле осуществляются переход $A P^{+} \rightarrow A P^{-}$с промежуточным переходом $P^{-} \rightarrow A P^{-}$. Таким образом для наблюдения немонотонной релаксации необходимо чтобы поля этих переходов совпадали. Для определения полей переходов запишем полные энергии стабильных состояний намагниченности, как суммы зеемановской и обменной энергии

$$
\begin{gathered}
E_{P^{+}}=-\left(M_{b}+M_{t}\right) H+E_{\mathrm{EX}}, \\
E_{A P^{+}}=-\left(M_{b}-M_{t}\right) H-E_{\mathrm{EX}}, \\
E_{A P^{-}}=\left(M_{b}-M_{t}\right) H-E_{\mathrm{EX}}, \\
E_{P^{-}}=\left(M_{b}+M_{t}\right) H+E_{\mathrm{EX}},
\end{gathered}
$$

где $M_{b}$ и $M_{t}$ - намагниченности нижнего и верхнего слоев $\mathrm{Co}, H-$ напряженность магнитного поля, $E_{\mathrm{EX}}-$ энергия обменного взаимодействия между ферромагнитными слоями.

Переход из состояния $A P^{+}$в $A P^{-}$возможен, если процесс идет с понижением энергии, т.е. в таком магнитном поле, когда $E_{A P^{-}}<E_{A P^{+}}$. Так как при переходе $A P^{+} \rightarrow A P^{-}$перемагничиваются оба слоя, энергия барьера перемагничивания $U_{A P^{+} \rightarrow A P^{-}}=E_{b}+E_{t}$, где $E_{b}(T)$ и $E_{t}(T)$ энергии барьеров нижнего и верхнего слоев. Условие данного перехода можно записать в виде неравенства $E_{A P^{+}} \geq E_{A P^{-}}+E_{b}+E_{t}$. Подставляя в это условие вместо энергий выражения (2) и (3), получим

$$
-\left(M_{b}-M_{t}\right) H-E_{\mathrm{EX}}=\left(M_{b}-M_{t}\right) H-E_{\mathrm{EX}}+E_{b}+E_{t} .
$$

Выразив значение $H$ из (5), получим значение поля переключения из состояния $A P^{+}$в $A P^{-}$:

$$
H_{A P^{+} \rightarrow A P^{-}}=-1 / 2\left(E_{b}+E_{t}\right) /\left(M_{b}-M_{t}\right) .
$$

Этот переход может быть монотонным, если критическое поле не совпадает с критическим полем другого перехода, а может быть немонотонным, при совпадении переходов. Запишем теперь условия перехода $P^{-} \rightarrow A P^{-}$ из уравнений (2) и (4) с учетом потенциального барьера между этими состояниями. Так как при данном переходе перемагничивается только нижний ферромагнитный слой, потенциальный барьер $U_{P^{-} \rightarrow A P^{-}}=E_{b}$.

$$
\left(M_{b}+M_{t}\right) H+E_{\mathrm{EX}}=\left(M_{b}-M_{t}\right) H-E_{\mathrm{EX}}+E_{b} .
$$

По аналогии с (6) из (7) поле перехода $P^{-} \rightarrow A P^{-}$равно

$$
H_{P^{-} \rightarrow A P^{-}}=-\left(2 E_{\mathrm{EX}}-E_{t}\right) / 2 M_{t} .
$$

Выражения (6) и (8) описывают критические поля переключения. Приравнивая уравнения (6) и (8) между собой, получаем

$$
E_{t}=2 E_{\mathrm{EX}}\left(1-\left(M_{t} / M_{b}\right)\right)-E_{b}\left(M_{t} / M_{b} .\right.
$$


Полученное условие немонотонной релаксации (9) не связано с ее дальнейшим развитием во времени, а является критерием ее страта, определяемым лишь балансом упомянутых энергий.

В образце, обсуждаемом в данной статье, перемагничивание происходит путем движения доменной стенки. Энергия барьера доменной стенки зависит от ее поля закрепления, которое определяется путем аппроксимации полевой зависимости скорости движения доменной стенки в режиме ползучести (рис. 5) формулой

$$
v_{D W}=v_{0} \exp \left(\left(H-H_{P}\right) / H_{f}\right),
$$

где $v_{0}$ - характерная скорость доменной стенки, $H-$ движущее постоянное поле, направленное перпендикулярно поверхности образца, $H_{P}-$ поле закрепления доменной стенки, $H_{f}-$ флуктуационное поле. Значение поля закрепления, при $T=300 \mathrm{~K}$ для доменной границы $A P^{-}$при переходе $A P^{+} \rightarrow A P^{-}$, составляет $H_{P}=-272 \mathrm{Oе}$, что соответствует критическому полю переключения из состояния $A P^{+}$в $A P^{-}$, полученному из полевой зависимости намагниченности при тех же условиях. Таким образом, барьерные энергии двух ФМ-слоев, определяемые из критических полей переключения хоть зависят от механизма релаксации намагниченности, но экспериментально определяются одинаково для всех механизмов перемагничивания.

Описанные ранее в [13] результаты свидетельствуют о вкладе взаимодействия Дзялошинского-Мория в энергию состояния $P^{-}$. С учетом энергии этого взаимодействия уравнение (9) принимает вид

$$
\begin{aligned}
E_{t}=2 E_{\mathrm{EX}}(1 & \left.-\left(M_{t} / M_{b}\right)\right)-E_{\mathrm{DMI}}\left(1-\left(M_{t} / M_{b}\right)\right) \\
& -E_{b}\left(M_{t} / M_{b}\right),
\end{aligned}
$$

где $E_{\mathrm{DMI}}-$ энергия взаимодействия ДзялошинскогоМория. Наличие члена $-E_{\mathrm{DMI}}\left(1-\left(M_{t} / M_{b}\right)\right)$ в уравнении (11), приводит к плоскопараллельному переносу прямой (9). Значение этого переноса незначительно, ввиду малого значения $E_{\mathrm{DMI}}$ по сравнению с $E_{\mathrm{EX}}$.

На рис. 6 графически изображены экспериментальные значения $E_{t}$ и $E_{b}$ в интервале температур от 50 до $300 \mathrm{~K}$ - геометрическое место точек, удовлетворяющих релаксации. (Разумеется, не существует зависимости $E_{t}$ от $\left.E_{b}\right)$. Каждая точка указывает значения $E_{t}$ и $E_{b}$ при определенной температуре. Энергии барьеров перемагничивания $Ф$ М-слоев не связаны физически между собой, и зависят лишь от температуры и толщины ФМ-слоев.

Выражение (9) является условием немонотонной релаксации в СФ. Чтобы проверить предсказательную силу (9) мы сравнили значения энергий барьеров перемагничивания верхнего $E_{t}$ и нижнего $E_{b}$ слоев Co, полученных экспериментально из петли магнитного гистерезиса для $T=100 \mathrm{~K}$, со значениями $E_{t}$ и $E_{b}$ полученными из уравнения (9). Для этого в уравнение (9) мы подставили экспериментальные значения $M_{b}, M_{t}$ и

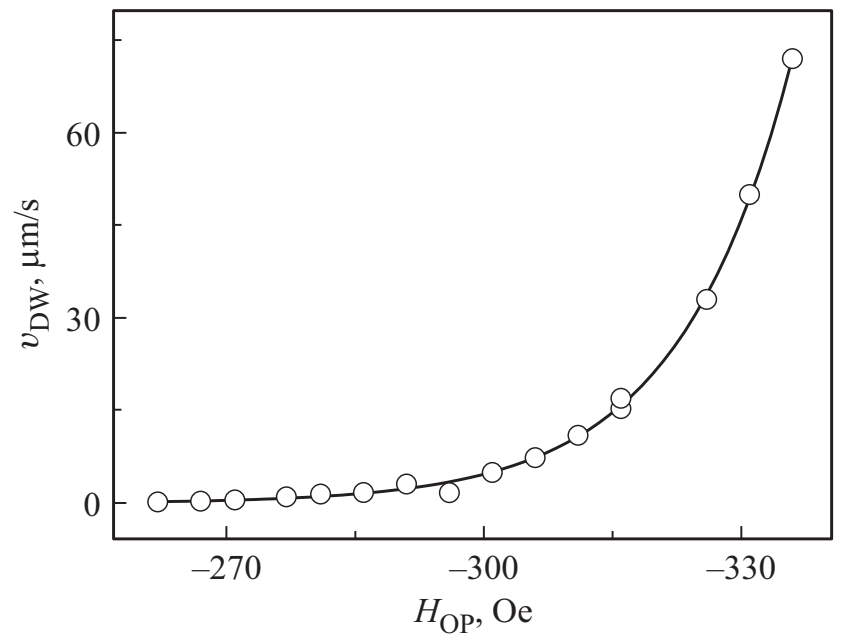

Рис. 5. Зависимость скорости доменной стенки $A P^{-}$при переходе $A P^{+} \rightarrow A P^{-}$от постоянного поля $H_{\mathrm{OP}}$, направленного перпендикулярно поверхности образца, при $T=300 \mathrm{~K}$. Сплошной линией показана аппроксимация уравнением (10).

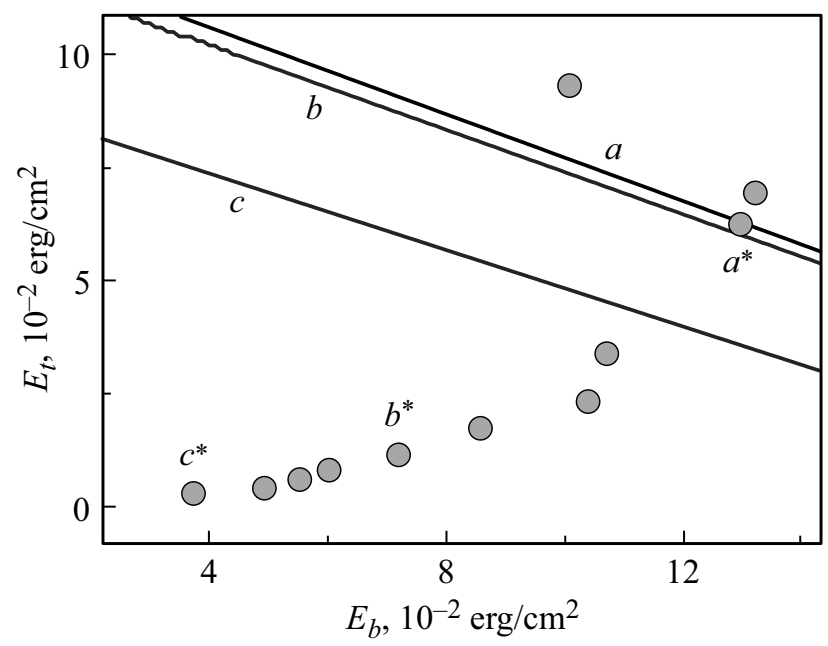

Рис. 6. Экспериментальные значения энергий барьеров перемагничивания верхнего $E_{t}$ и нижнего $E_{b}$ слоя Со. Каждая точка указывает значения $E_{t}$ и $E_{b}$ при определенной температуре. Сплошными линиями показаны условия, задаваемые уравнением (9) для экспериментальных значений $M_{b}, M_{t}$ и $E_{\mathrm{EX}}: a-$ при $T=100, b-$ при $T=200, c-$ при $T=300 \mathrm{~K}$. Соответствующие этим температурам реальные экспериментальные точки $E_{t}$ и $E_{b}$ при 100,200 и $300 \mathrm{~K}$ обозначены буквами $a^{*}, b^{*}$ и $c^{*}$ соответственно. Точка $a^{*}$ удовлетворяет условиям $E_{t}$ и $E_{b}$ немонотонной релаксации.

$E_{\mathrm{EX}}$ для $T=100 \mathrm{~K}$, и построили прямую $a$ (рис. 6). Из рис. 6 видно что эта теоретическая прямая $a$ пересекает экспериментальную точку $a^{*}$ для $T=100 \mathrm{~K}$. Чтобы удостовериться в том, что уравнение (9) описывает лишь условия немонотонной релаксации намагниченности, мы построили прямые $b$ и $c$ на рис. 6 подставляя в уравнение (9) экспериментальные значение $M_{b}, M_{t}$ и $E_{\mathrm{EX}}$ для 200 и $300 \mathrm{~K}$. Эти прямые $b$ и $c$ не пересекают 
экспериментальные точки $b^{*}$ и $c^{*}$ для 200 и $300 \mathrm{~K}$. Данная проверка проведена для всех температур, для которых имеются экспериментальные значения $E_{t}$ и $E_{b}$ (прямые не показаны на рис. 6). Ни одна прямая не пересекает экспериментальную точку для соответствующей температуры. Из этого можно сделать вывод что уравнение (9) является условием немонотонной релаксации, которое устанавливает связь между энергиями барьеров перемагничивания двух ферромагнитных слоев синтетического ферримагнетика. Физические причины существования ограничений на соотношение магнитных барьеров перемагничивания слоев, задаваемых (9), по-видимому, могут объясняться спецификой взаимодействия зародышей намагниченности разных типов в двух ферромагнитных слоях и требуют дальнейших исследований.

\section{4. Выводы}

Немонотонная релаксация намагниченности в синтетических ферримагнетиках с перпендикулярной магнитной анизотропией наблюдается при соотношении энергий барьеров перемагничивания в ферромагнитных слоях, обеспечивающем равенство полей переключение между несколькими типами переходов. Это соотношение описывает условие сосуществования трех типов зародышей намагниченности $A P^{+}, A P^{-}$и $P^{-}$. Зная температурные зависимости намагниченности, энергии барьеров ферромагнитных слоев СФ и их обменной энергии, можно достоверно предсказать толщину свободного слоя ферромагнетика и температуру, при которых будет наблюдаться немонотонная релаксация намагниченности.

\section{Благодарности}

Авторы благодарны профессору S. Mangin (Institut Jean Lamour, Université de Lorraine, Nancy, France) за предоставленные образцы и плодотворные дискуссии.

\section{Финансирование работы}

Работа выполнена при поддержке гранта 3.1992.2017/4.6 в рамках конкурса научных проектов, выполняемых научными коллективами исследовательских центров и(или) научных лабораторий образовательных организаций высшего образования. А.И. Безверхний поддержан грантом РФФИ в рамках научного проекта № 19-32-90128. Работа выполнена в рамках программы Топ5-100. Работа выполнена при поддержке гранта 2644.2020.2 Президента Российской Федерации для государственной поддержки ведущих научных школ.

\section{Конфликт интересов}

Авторы заявляют, что у них нет конфликта интересов.

\section{Список литературы}

[1] T. Fache, H.S. Tarazona, J. Liu, G. L'vova, M.J. Applegate, J.C. Rojas-Sanchez, S. Petit-Waletot, C.V. Landauro, QuispeMarcatoma, R. Morgunov, C.H.W. Barnes, S. Mangin. Phys. Rev. B 98, 064410 (2018).

[2] Р.Б. Моргунов, Г.Л. Львова. Письма в ЖЭТФ 108, 124 (2018).

[3] P.F. Carcia, M. Reilly, Z.G. Li. IEEE Trans. Magn. 30, 4395 (1994).

[4] A. Talantsev, Y. Lu, T. Fache, M. Lavantant, A. Hamadeh, A. Aristov, O. Koplak, R. Morgunov, S. Mangin. J. Phys.: Condens. Matter 30, 135804 (2018).

[5] N. Nakajima, T. Koide, T. Shirada, H. Miyauchi, H. Fukutani, A. Fujimori, K. Iio, T. Katayama, M. Nývlt, Y. Suzuki. Phys. Rev. Lett. 81, 5229 (1998).

[6] R. Morgunov, A. Hamadeh, T. Fache, G. Lvova, O. Koplak, A. Talantsev, S. Mangin. Superlat. Microstruct. 104, 509 (2017).

[7] R.B. Morgunov, E.I. Kunitsyna, A.D. Talantsev, O.V. Koplak, T. Fache, Y. Lu, S. Mangin. APL 114, 222402 (2019).

[8] K. Shahbazi, J.-V. Kim, H.T. Nembach, J.M. Shaw, A. Bischof, M.D. Rossell, V. Jeudy, T.A. Moore, C.H. Marrows. Phys. Rev. B 99, 094409 (2019).

[9] A. Hrabec, N.A. Porter, A. Wells, M.J. Beniez, G. Burnell, S. McVitie, D. McGrouther, T.A. Moore, C.H. Marrows. Phys. Rev. B 90, 020402(R) (2014).

[10] R.B. Morgunov, G.L. L'vova, A.D. Talantsev, O.V. Koplak, T. Fache, S. Mangin. J. Magn. Magn. Mater. 459, 33 (2018).

[11] E. Fatuzzo. Phys. Rev. 127, 1999 (1962).

[12] M. Labrune, S. Andrieu, F. Rio, P. Bernstein. J. Magn. Magn. Mater. 80, 211 (1989).

[13] R.B. Morgunov, A.V. Yurov, V.A. Yurov, A.D. Talantsev, A.I. Bezverhnii, O.V. Koplak. Phys. Rev. B 100, 144407 (2019).

Редактор Д.В. Жуманов 